\title{
Retrorectal Cystic Hamartoma with Malignant Transformation
}

\author{
Andreia Cruz ${ }^{1}$, Sarah Lopes ${ }^{1}$, Maria Leitão¹, Sónia Carvalho², Olga Sousa ${ }^{3}$, \\ Manuela Machado ${ }^{*}$
}

${ }^{1}$ Medical Oncology Department, Portuguese Institute of Oncology, IPO Porto, Portugal

${ }^{2}$ Pathology Department, IPO Porto, Portugal

${ }^{3}$ Radiotherapy Department, IPO Porto, Portugal

Email: *m.machado.fn@gmail.com

How to cite this paper: Cruz, A., Lopes, S., Leitão, M., Carvalho, S., Sousa, O. and Machado, M. (2020) Retrorectal Cystic Hamartoma with Malignant Transformation. Journal of Cancer Therapy, 11, 18-25. https://doi.org/10.4236/jct.2020.111002

Received: December 10, 2019

Accepted: January 13, 2020

Published: January 16, 2020

Copyright $\odot 2020$ by author(s) and Scientific Research Publishing Inc. This work is licensed under the Creative Commons Attribution International License (CC BY 4.0).

http://creativecommons.org/licenses/by/4.0/ (c) (i) Open Access

\begin{abstract}
Retrorectal cystic hamartomas are rare congenital lesions that can undergo malignant transformation, and adenocarcinoma is the most frequently described histological type. The authors describe a case of a 53-year-old female patient with a localized well-differentiated adenocarcinoma that developed in a retrorectal cystic hamartoma. The patient was submitted to surgery (a Kraske procedure), with an R1 resection, followed by adjuvant radio-chemotherapy. After 23 months of follow up, the patient remains free from disease recurrence. Given the rarity of this entity, this case allows us to reflect on the differential diagnosis, therapeutic approach and patients' follow-up.
\end{abstract}

\section{Keywords}

Retrorectal Cystic Hamartoma, Tailgut Cyst, Presacral, Malignant Transformation, Adenocarcinoma

\section{Introduction}

Retrorectal cystic hamartomas ( $\mathrm{RCH})$, or tailgut cysts, are rare congenital presacral lesions that arise from an aberrant remnant of postnatal hindgut due to incomplete involution during embryological development [1]. According to the literature, malignant transformation of the epithelial or stromal components of $\mathrm{RCH}$ may occur in up to $14 \%$ of patients [2]. Adenocarcinoma is the most frequently described histological type, followed by carcinoid tumors [2] [3] [4] [5].

It is most common in middle-aged women and usually is discovered incidentally or on the study of pain, rectal bleeding, or symptoms related to mass effect on the rectum or bladder [6]. Magnetic resonance imaging (MRI) is the most use- 
ful exam for diagnosis and preoperative planning [7]. Biopsy should be performed in unresectable or locally advanced cases, in order to define the best therapeutic strategy [2].

Complete surgical resection is the standard treatment if possible [3]. Systemic treatment depends on histology and stage. Adjuvant radio-chemotherapy and palliative chemotherapy including 5-fluorouracil and leucovorin have been reported for adenocarcinomas [8] [9] [10] [11].

Prognosis for $\mathrm{RCH}$ with malignant transformation depends on complete surgical resection, tumour histology and grade, with local recurrences and distant metastasis occasionally reported [12]. Follow-up is clinical, with imaging according to clinical history and examination [12].

The authors describe a case of a 53-year-old female patient with a localized well-differentiated adenocarcinoma that developed in a retrorectal cystic hamartoma, after obtaining her consent. Given the rarity of this entity, we aimed to reflect on questions such as the differential diagnosis, therapeutic approach and patients' follow-up.

\section{Case Report}

A 53-year-old female patient presented with a history of a few weeks of pelvic pain, in May 2017. She had no relevant comorbidities. Digital rectal examination revealed a fixed, hard, posterior mass at $4-5 \mathrm{~cm}$ of the anal margin, with normal mucosa. No other relevant findings on the physical exam were present.

A pelvic MRI showed an oval lesion, in the pre-sacral and right para-rectal area, with lobulated contours, measuring $5 \times 3 \times 3.5 \mathrm{~cm}$, with heterogeneous signal intensity, and with preservation of the adipose plane with the rectal ampulla (Figure 1). The colonoscopy showed a bulge in the rectum, at $7-11 \mathrm{~cm}$ from the anal margin, covered by normal mucosa, suggestive of extrinsic compression (Figure 2). A thoraco-abdominopelvic computed tomography (CT) was performed and did not show any evidence of suspicious lymph nodes or distant metastasis. Carcinoembryonic antigen (CEA) wasn't elevated. The histological exam, of a CT-guided biopsy, reported intestinal-type adenocarcinoma structures imbedded in desmoplastic fibrous connective tissue. A positron-emission tomography-CT (PET-CT) showed the para-rectal lesion with a SUV max of 11.8, with no other areas of $18-F D G$ fixation.

The patient underwent a Kraske procedure with an en bloc segmental rectal resection (Figure 3), on December $5^{\text {th }}, 2017$. The invasion of S4 and S5 was reported intraoperatively. The postoperative period occured without complications. The histological result confirmed an intestinal-type adenocarcinoma, co-existing with a multiloculated cystic lesion covered by columnar epithelium with areas of lowand high grades dysplasia, with extensive lymphovascular and perineural permeation, and marked rupture phenomena (Figure 4). Infiltration of adjacent soft tissues and sacrum (focally) was present but without invasion of the rectum wall. The upper and anterior soft tissues margins were positive for tumour involvement. 


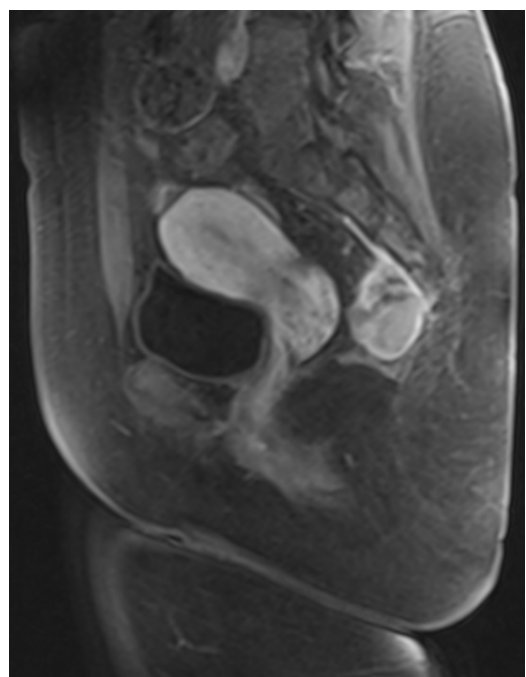

(a)

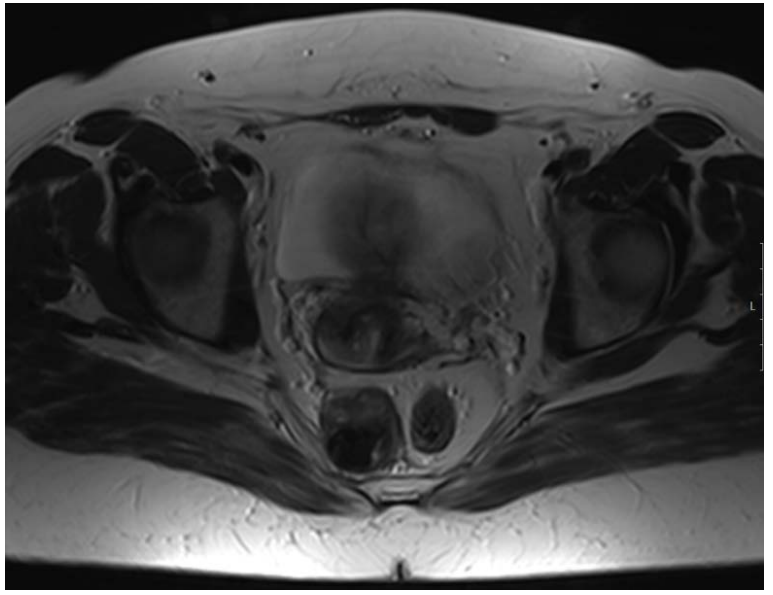

(b)

Figure 1. Pelvic MRI showing an oval lesion, in the pre-sacral and right para-rectal area (T1 sequence with venous contrast (a)), with lobulated contours, measuring $5 \times 3 \times 3.5$ $\mathrm{cm}$, with heterogeneous signal intensity, and with preservation of the adipose plane with the rectal ampulla (T2 sequence (b)).

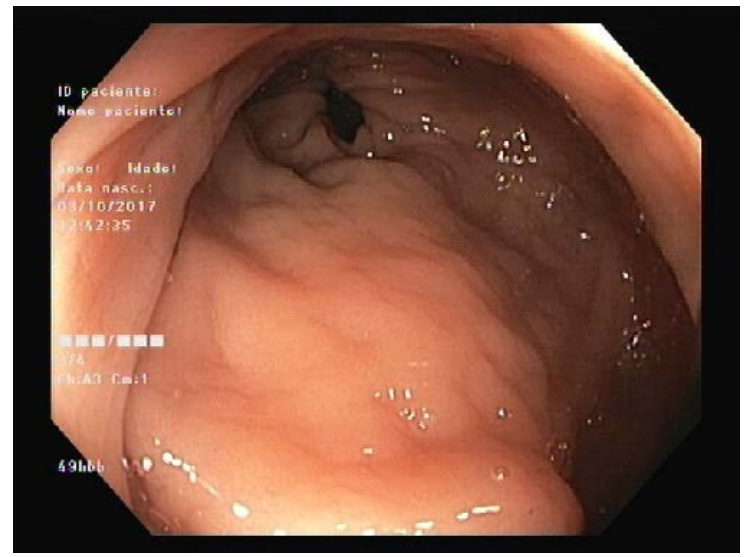

Figure 2. Colonoscopy showing a bulge in the rectum, at $7-11 \mathrm{~cm}$ from the anal margin, covered by normal mucosa, suggestive of extrinsic compression. 


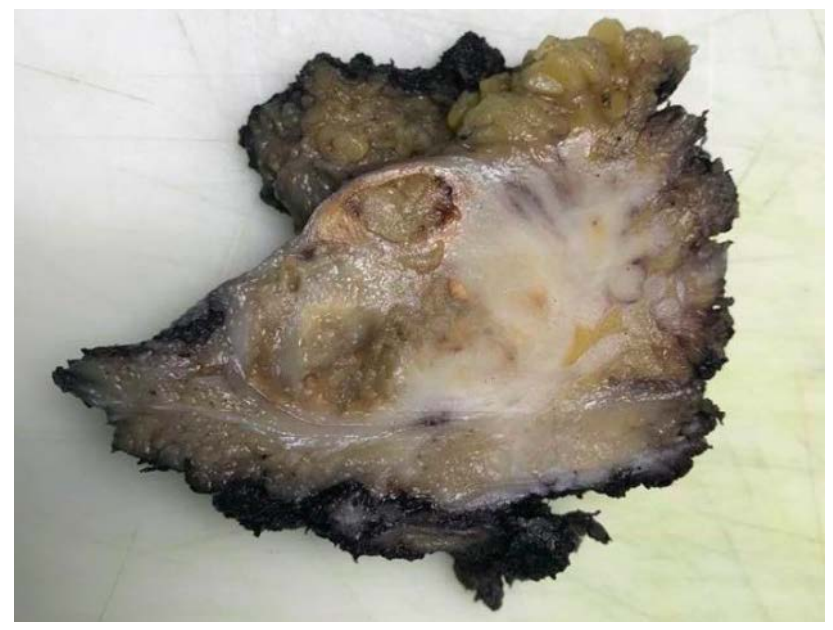

Figure 3. Macroscopic image of a predominantly whitish and hardened neoplasm, with $4.9 \times 4 \times 3 \mathrm{~cm}$, containing a cavitated area, multiloculated and with brownish content, with $2.5 \mathrm{~cm}$.

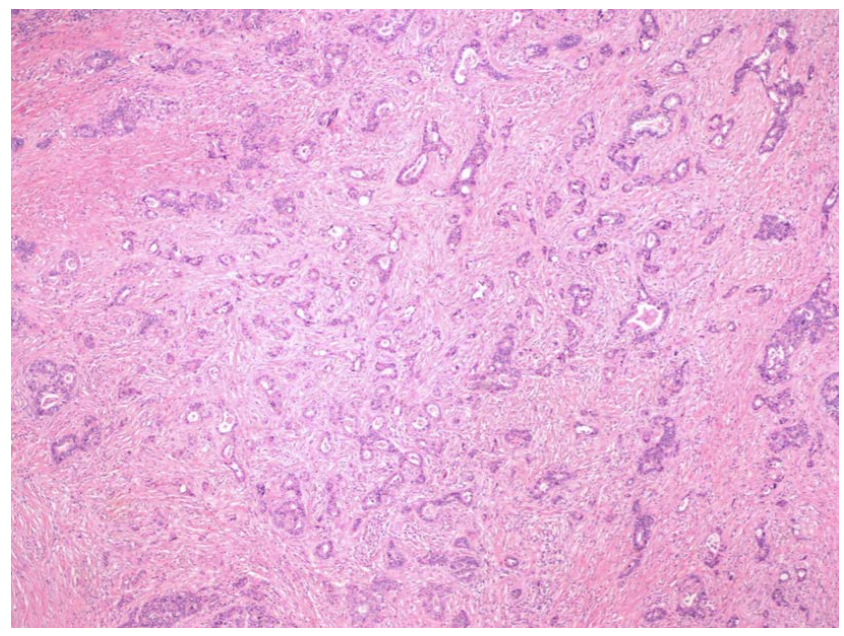

(a)

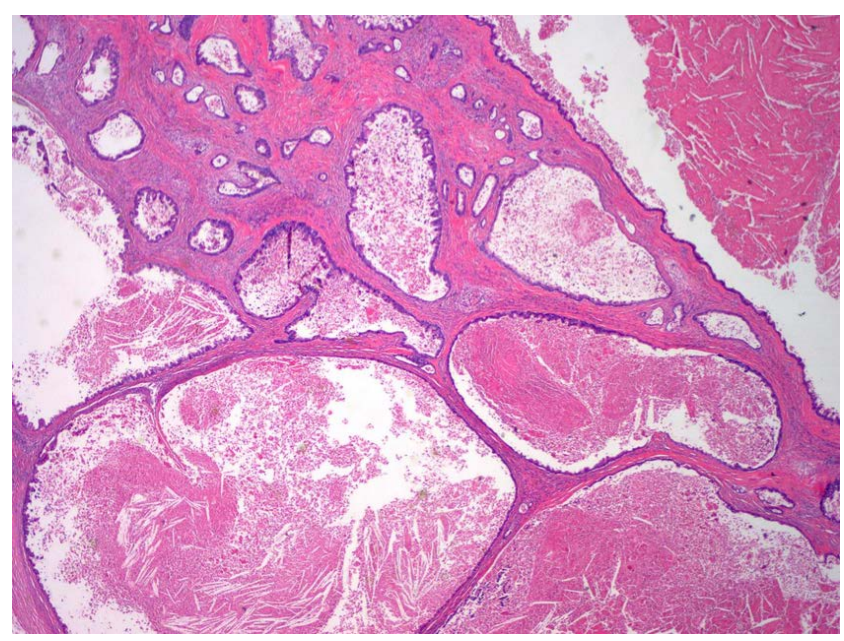

(b)

Figure 4. Histological result confirming an intestinal-type adenocarcinoma (a), co-existing with a multiloculated cystic lesion (b). 
Three lymph nodes were isolated and none of them were metastasized. The histological evaluation concluded that the tumour was a well-differentiated adenocarcinoma that developed in a RCH. Two months after surgery, a pelvic MRI and PET-CT were repeated, and no images were suspicious of persistence/recurrence of disease.

The patient subsequently received adjuvant radiotherapy (45 Gy to the regional lymph nodes and 54 Gy to the surgical bed and sacrum, 1.8 Gy per fraction, through Intensity Modulated Radiation Therapy, Figure 5) with concomitant chemotherapy (capecitabine $825 \mathrm{mg} / \mathrm{m}^{2} \mathrm{q} 12 \mathrm{~h}$ ), between March $20^{\text {th }}$ and April $27^{\text {th }} 2018$, without interruptions and with no record of relevant toxicities.

Follow-up has been performed with clinical evaluation and pelvic MRI every three to four months, with no evidence of disease recurrence, 23 months after surgery.

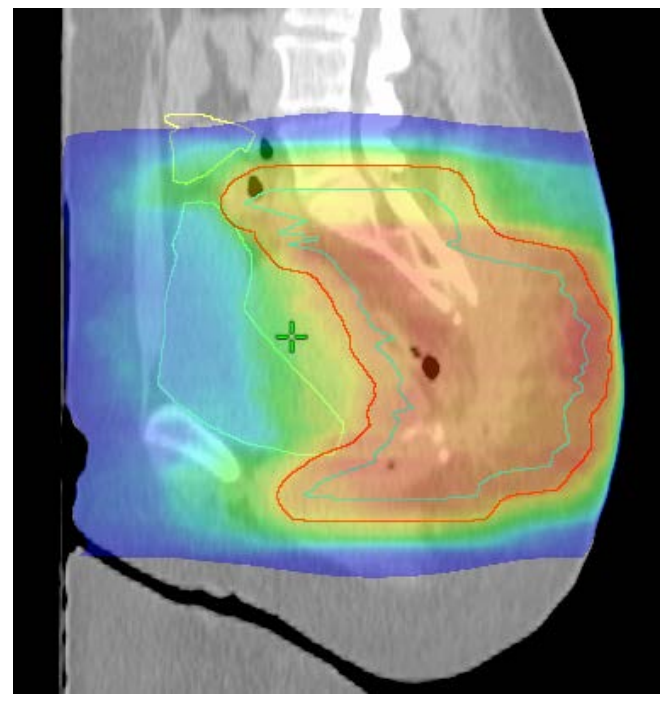

(a)

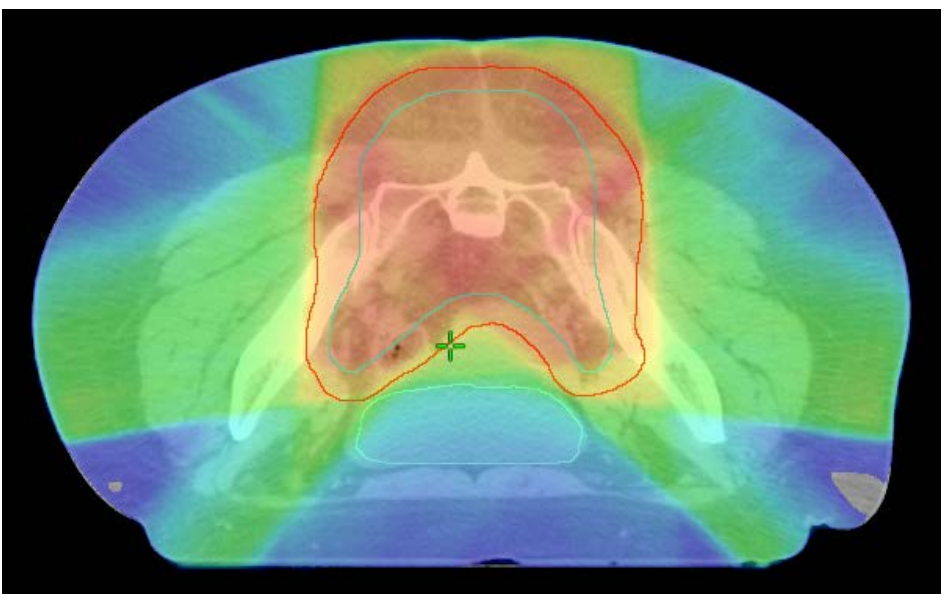

(b)

Figure 5. Radiotherapy planning in the sagittal (6) and axial plan (7), to give 45 Gy to the regional lymph nodes and 54 Gy to the surgical bed and sacrum, 1.8 Gy per fraction, through Intensity Modulated Radiation Therapy. 


\section{Discussion}

$\mathrm{RCH}$ have an estimated overall incidence of 1 in 40,000, but more frequent imaging exams and advances in diagnostic techniques are leading to an increased identification of cases [13] [14].

Malignant transformation of $\mathrm{RCH}$ to adenocarcinoma was first described in 1931 [15]. Although rare, malignancy was described in up to $14 \%$ of patients with $\mathrm{RCH}$, enhancing the importance of the inclusion of this entity in the differential diagnosis of a retrorectal mass [2]. These lesions should be distinguished from others which may occur in that localization, such as teratomas, epidermal cysts, rectal duplication cysts, anal gland cysts and anal gland carcinomas.

MRI is the most helpful investigation exam, with a diagnostic accuracy of $100 \%$ in defining the lesion, and a reported positive predictive value of $100 \%$ for malignancy [12]. The preoperative biopsy for RCH is controversial, especially with the improvement in the imaging tools and also due to biopsy-related complications such as infection, hematoma, and needle track implants [16]. However, in this case where the concern about malignant degeneration was high, the advantage was that it could help with optimal planning of surgical approach and adjuvant therapy, and also in the information about prognosis.

Complete surgical excision is the therapy of choice and the most common surgical approach described is via a posterior para-sacral incision, particularly for lesions below the level of S3 or the sacral promontory [6]. This approach is safe and is associated with low risk of local recurrence, although fistulas to the rectum may be more common than in others situations [3]. Unfortunately, in this case surgery was R1, as the upper and anterior margins of the soft tissues were positive. The sacral bone margins were not involved, which reinforces the relevance of the trans-sacral resection. Attending to histological type and localization of these tumours, we should debate if there is a place for preoperative radio-chemotherapy to improve R0 resections, in analogy to what is performed in rectal cancers.

Because of the small number of patients with malignancy in published series of $\mathrm{RCH}$, no significant conclusions can be drawn regarding local or distant recurrence [3]. According to histology and R1 surgery, our patient was proposed for complementary treatment with radio-chemotherapy, remaining without evidence of disease after approximately two years of follow-up. Nevertheless, the patient is being closely monitored, clinically and with radiological exams. In case of unresectable local recurrence or distant metastasis, and although only palliative chemotherapy including 5-fluorouracil and leucovorin have been reported, maybe we should consider association regimens, such as with oxaliplatin or irinotecan, according to what is done, nowadays, in colorectal tumours.

\section{Conclusion}

$\mathrm{RCH}$ with malignant transformation should be considered in the differential diagnosis of a retrorectal mass. After a trimodal treatment, our patient is being closely 
monitored and remains free from disease recurrence for almost two years. This case reinforces the importance of the multidisciplinary approach in such challenging situations.

\section{Acknowledgements}

Radiology Department of IPO Porto, by providing the MRI images.

\section{Conflicts of Interest}

The authors declare no conflicts of interest regarding the publication of this paper.

\section{References}

[1] Patsouras, D., Pawa, N., Osmani, H., et al. (2015) Management of Tailgut Cysts in a Tertiary Referral Centre: A 10-Year Experience. Colorectal Disease, 17, 724-729. https://doi.org/10.1111/codi.12919

[2] Akbulut, S. (2013) Unusual Cause of Defecation Disturbance: A Presacral Tailgut Cyst. European Review for Medical and Pharmacological Sciences, 17, 1688-1699.

[3] Mathis, K.L., Dozois, E.J., Grewal, M.S., et al. (2010) Malignant Risk and Surgical Outcomes of Presacral Tailgut Cysts. British Journal of Surgery, 97, 575-579. https://doi.org/10.1002/bjs.6915

[4] Horenstein, M.G., Erlandson, R.A., Gonzalez-Cueto, D.M., et al. (1998) Presacral Carcinoid Tumors: Report of Three Cases and Review of the Literature. The American Journal of Surgical Pathology, 22, 251-255. https://doi.org/10.1097/00000478-199802000-00015

[5] Prasad, A.R., Amin, M.B., Randolph, T.L., et al. (2000) Retrorectal Cystic Hamartoma Report of 5 Cases with Malignancy Arising in 2. Archives of Pathology \& Laboratory Medicine, 124, 725-729.

[6] Hufkens, A.S., Cools, P. and Leyman, P. (2019) Tailgut Cyst: Report of Three Cases and Review of the Literature. Acta Chirurgica Belgica, 119, 110-117. https://doi.org/10.1080/00015458.2017.1353758

[7] Shetty, A.S., Loch, R., Yoo, N., et al. (2015) Imaging of Tailgut Cysts. Abdominal Radiology, 40, 2783-2795. https://doi.org/10.1007/s00261-015-0463-3

[8] Jarboui, S., Jarraya, H., Mihoub, M.B., et al. (2008) Retrorectal Cystic Hamartoma Associated with Malignant Disease. Canadian Journal of Surgery, 51, E115-E116.

[9] Van Roggen, J.F.G., Welvaart, K., de Roos, A., et al. (1999) Adenocarcinoma Arising within a Tailgut Cyst: Clinicopathological Description and Follow up of an Unusual Case. Journal of Clinical Pathology, 52, 310-312.

https://doi.org/10.1136/jcp.52.4.310

[10] Zappa, L., Godwin, T.A. and Sugarbaker, P.H. (2009) Tailgut Cyst, an Unusual Cause of Pseudomyxoma Peritonei. Tumori, 95, 514-517. https://doi.org/10.1177/030089160909500418

[11] Tampi, C., Lotwala, V., Lakdawala, M., et al. (2007) Case Report Retrorectal Cyst Hamartoma (Tailgut Cyst) with Malignant Transformation. Gynecologic Oncology, 105, 266-268. https://doi.org/10.1016/j.ygyno.2007.01.008

[12] Chereau, N., Lefevre, J.H., Meurette, G., et al. (2013) Surgical Resection of Retrorectal Tumours in Adults: Long-Term Results in 47 Patients. Colorectal Disease, 15, e476-e482. https://doi.org/10.1111/codi.12255 
[13] Jao, S.W., Beart, R.W., Spencer, R.J., et al. (1985) Retrorectaltumors Mayo Clinic Experience, 1960-1979. Diseases of the Colon \& Rectum, 28, 644-652. https://doi.org/10.1007/BF02553440

[14] Hjermstad, B.M. and Helwig, W.B. (1988) Tailgut Cysts. American Journal of Clinical Pathology, 89, 139-147. https://doi.org/10.1093/ajcp/89.2.139

[15] Ballantine, E.N. (1931) Sacrococcygeal Tumours. Adenocarcinoma of a Cystic Congenital Embryonal Remnant. Arch Pathology, 14, 1-9.

[16] Bathla, L., Singh, L. and Agarwal, P.N. (2013) Retrorectal Cystic Hamartoma (Tailgut Cyst): Report of a Case and Review of Literature. Indian Journal of Surgery, 75, S204-S207. https://doi.org/10.1007/s12262-012-0633-2 